\title{
Signal focusing through active transport
}

\author{
Aljaž Godec ${ }^{1,2}$ and Ralf Metzler ${ }^{1,3}$ \\ ${ }^{1}$ Institute of Physics \& Astronomy, University of Potsdam, D-14476 Potsdam-Golm, Germany \\ ${ }^{2}$ Laboratory for Molecular Modeling, National Institute of Chemistry, Hajdrihova 19, 1000 Ljubljana, Slovenia \\ ${ }^{3}$ Department of Physics, Tampere University of Technology, FI-33101 Tampere, Finland
}

(Received 13 January 2015; published 2 July 2015)

\begin{abstract}
The accuracy of molecular signaling in biological cells and novel diagnostic devices is ultimately limited by the counting noise floor imposed by the thermal diffusion. Motivated by the fact that messenger RNA and vesicle-engulfed signaling molecules transiently bind to molecular motors and are actively transported in biological cells, we show here that the random active delivery of signaling particles to within a typical diffusion distance to the receptor generically reduces the correlation time of the counting noise. Considering a variety of signaling particle sizes from mRNA to vesicles and cell sizes from prokaryotic to eukaryotic cells, we show that the conditions for active focusing — faster and more precise signaling-are indeed compatible with observations in living cells. Our results improve the understanding of molecular cellular signaling and novel diagnostic devices.
\end{abstract}

DOI: 10.1103/PhysRevE.92.010701

PACS number(s): 87.15.Ya, 87.15.Vv, 87.16.Xa

\section{INTRODUCTION}

Cellular signaling relaying external or internal biochemical cues typically involves low copy numbers of messenger molecules, inevitably effecting appreciable fluctuations in the count of molecular binding events at specific receptors [1-12]. A similar limitation by counting noise is encountered in recent microscopic diagnostic devices to which sensitivity is a key factor [13]. Modern microscopic techniques reveal molecular signaling events and underline their inherent stochasticity in living cells [14-17]. Nevertheless, molecular signaling pathways in biological cells operate at remarkable precision [18,19].

The first heuristic argument about noise limitation to biological concentration measurements is due to Berg and Purcell (BP) assuming biochemical receptors to count the number $N$ of specific molecules in a volume equal to their linear dimension $a$ [2]; $N$ is then limited by Poissonian noise $\delta N \sim$ $\langle N\rangle^{1 / 2}$. The time between two independent measurements is set by the time $\tau_{D} \sim a^{2} / D$ needed to clear the volume by diffusion, $D$ being the molecular diffusivity. Averaging over a time $\tau_{m}$ thus allows $N_{m} \sim \tau_{m} / \tau_{D}$ independent measurements, reducing the noise by the factor $N_{m}^{1 / 2}$. The relative accuracy to measure a background concentration $\langle c\rangle$ is thus $\overline{\delta c^{2}} /\langle c\rangle^{2} \sim$ $\left(D a\langle c\rangle \tau_{m}\right)^{-1}[1,2]$. When the additional binding dynamics to the biochemical receptor is explicitly taken into account, this relative error becomes [1]

$$
\frac{\overline{\delta c^{2}}}{\langle c\rangle^{2}} \simeq \frac{2}{k_{\mathrm{on}}\langle c\rangle(1-\langle n\rangle) \tau_{m}}+\frac{1}{\pi D a\langle c\rangle \tau_{m}} .
$$

The first term stems from the Markovian binding (or unbinding) to the receptor at detailed balance conditions with binding rate $k_{\text {on }}$ and mean receptor occupancy $\langle n\rangle$. The second term is the diffusional noise, identical to the result by BP up to a prefactor $[2(1-\langle n\rangle)]^{-1}$ important for $\langle n\rangle \simeq 1[2,11]$. An alternative approach reproducing the Berg-Purcell prefactor is used in Ref. [11]. Both methods [1,11] rest on a mean-field approximation but differ in the treatment of correlations between mean concentrations of free and bound molecules (see Ref. [20]). Inspired by the ideas of BP, several studies unraveled the crucial role of diffusional noise in biochemical signaling [3-5,7-10,12]. Experiments suggest that cells indeed operate very close to the fundamental accuracy limit [1].

Here we extend the result (1) to the case when the signaling molecules are not only freely diffusing in the cell but actively transported along cellular filaments by motor proteins $[21,22]$ effecting intermittent ballistic excursions [23-26]. Such an additional active component occurs when extracellular signaling molecules are taken up by the cell via endocytosis: The molecules are engulfed into submicron lipid vesicles and then intermittently transported through the cell by motors [27]. A similar combination of free diffusion and active transport occurs when virus particles invade a living cell [28]. However, even free molecules such as messenger RNA may attach to motors [29] or proteins move in a directed fashion due to cytoplasmic drag [30]. To incorporate the active component we employ the theory of a random intermittent search for hidden targets [31], which was recently used to analyze reaction kinetics in active media [32]. We show that active transport enables both faster and more accurate sensing: An active noise floor exists, but it can be significantly lower than the purely diffusive counting noise (1). This active focusing reduces the noise correlation time and enables the receptor to more accurately detect relative concentration changes. Our model complements the existing works describing cellular signaling phenomena in active fluids [33] and has a direct impact on the design of active components in microscopic synthetic diagnostic systems based on molecular signals [13].

\section{MODEL}

We consider a signaling particle (vesicle, virus, mRNA, or protein) in three-dimensional cellular space, randomly switching between a passive diffusion phase $p$ with diffusivity $D$ and an active ballistic phase $a$ with velocity $\mathbf{v}(\Omega)$ of constant magnitude $v=|\mathbf{v}|$ [34] in the direction of the solid angle $\Omega$ following a Markovian dynamics (Fig. 1). Assuming ideally disordered cytoskeletal filament orientations, the spatial direction of active motion events is uniformly distributed. The duration of active and passive phases is exponentially distributed with mean $\tau_{a, p}$. The concentrations of freely diffusing and motor-bound signaling particles are $c_{p}(\mathbf{r}, t)$ and 


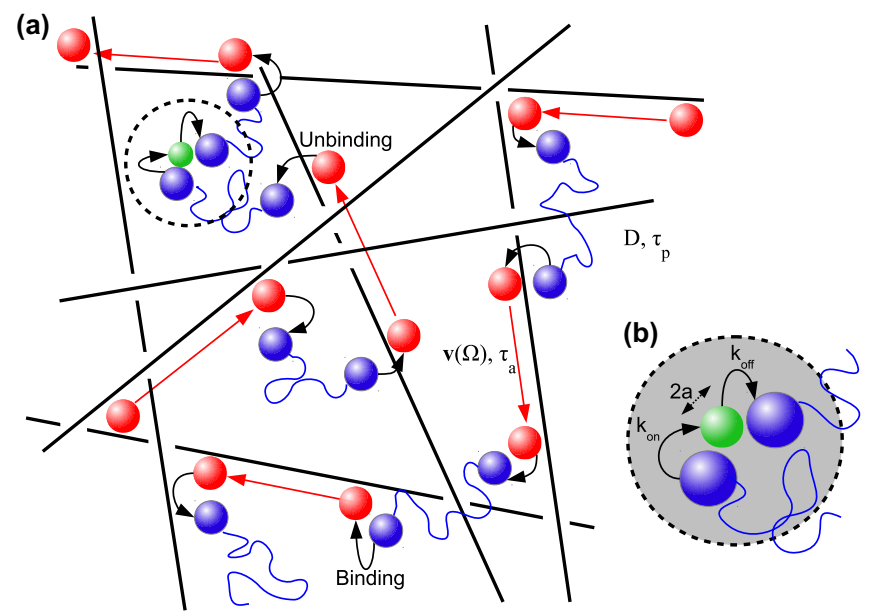

FIG. 1. (Color online) Model system. (a) Signaling particles perform passive thermal diffusion (blue phases) interrupted by active ballistic excursions with constant speed and random direction (red phases moving along the black motor tracks). The duration of both phases is distributed exponentially with mean times $\tau_{p, a}$. When the particle reaches the receptor (green sphere) it binds and dissociates with rates $k_{\text {on }}$ and $k_{\text {off }}$, respectively. (b) Receptor region.

$c_{a}(\mathbf{r}, \Omega, t)$. A receptor with radius $a$ is placed at $\mathbf{r}_{0}$. Then the fractional occupancy $n(t)$ of the receptor by a signaling particle evolves according to a mean-field kinetic scheme obeying detailed balance with on-off rates $k_{\text {on, off }}$,

$$
d n(t) / d t=k_{\mathrm{on}} c_{p}\left(\mathbf{r}_{0}, t\right)[1-n(t)]-k_{\mathrm{off}} n(t) .
$$

Assuming that the particle binds (unbinds) to (from) the receptor only from (to) the passive mode [32], the coupled set of equations for the concentrations $c_{a, p}$ reads

$$
\begin{aligned}
\frac{\partial c_{p}(\mathbf{r}, t)}{\partial t} & =D \nabla^{2} c_{p}+\int \frac{c_{a}}{\tau_{a}} d \Omega-\frac{c_{p}}{\tau_{p}}-\delta\left(\mathbf{r}-\mathbf{r}_{0}\right) \frac{d n(t)}{d t}, \\
\frac{\partial c_{a}(\mathbf{r}, \Omega, t)}{\partial t} & =-\nabla_{\mathbf{r}} \cdot\left[\mathbf{v}(\Omega) c_{a}\right]-\frac{c_{a}}{\tau_{a}}+\frac{c_{p}}{4 \pi \tau_{p}} .
\end{aligned}
$$

Signaling typically occurs in two stages. Initially a change in the concentration of signaling particles occurs either by exchange with the extracellular space $[19,35]$ or by variation of the production and/or degradation rates. Upon reequilibration (assumed to be much faster than the measurement time $\tau_{m}$ ) the receptor then reads out the concentration over the time $\tau_{m}$ in the measurement phase. In diagnostic devices equivalent phases will be observed after sample immersion and during detection. In an optimal setup equilibration should be as fast and the measurement should be as precise as possible. We now quantify the speed and precision of the two phases.

\section{SPEED}

We assume that the system equilibrates on a time scale over which the signaling molecules move a distance $L$ of the order of the cell size (or that of a cellular compartment). At this stage we neglect the analyte-receptor binding dynamics and adopt a probabilistic view of Eq. (3). The equilibration time $\tau_{i}$ is then defined by the mean-square displacement (MSD) $\left\langle\left|\mathbf{r}\left(\tau_{i}\right)\right|^{2}\right\rangle=$ $L^{2}$. Laplace and Fourier transforms of Eq. (3) with respect to time and space $[(\mathbf{r}, t) \rightarrow(\mathbf{k}, s)]$ allow a formal solution for $\tilde{c}_{a}$ and $\tilde{c}_{p}$. The MSD for a particle starting at the origin in the passive phase is obtained from $\left\langle\left|\mathbf{r}^{2}(s)\right|\right\rangle=-\nabla_{\mathbf{k}}^{2}\left(\int \tilde{c}_{a} d \Omega+\right.$ $\left.\tilde{c}_{p}\right)\left.\right|_{\mathbf{k}=0}$. Interchanging differentiation with respect to $\mathbf{k}$ and integration with respect to $\Omega$,

$$
\left\langle\left|\mathbf{r}^{2}(s)\right|\right\rangle=\frac{6 D\left[1+\tau_{p}^{-1} /\left(s+\tau_{a}^{-1}\right)\right]+2\left(v^{2} / \tau_{p}\right)\left[\left(s+\tau_{a}^{-1}+\tau_{p}^{-1}\right) /\left(s+\tau_{a}^{-1}\right)^{3}\right]}{s+\tau_{p}^{-1}-\left(\tau_{a} \tau_{p}\right)^{-1} /\left(s+\tau_{a}^{-1}\right)} .
$$

Laplace inversion leads to the MSD

$$
\left\langle\left|\mathbf{r}^{2}(t)\right|\right\rangle=2\left\{\left(v \tau_{a}\right)^{2} e^{-t / \tau_{a}}-\frac{v^{2}+3 D \tau_{p}^{-1}}{\left(\tau_{a}^{-1}+\tau_{p}^{-1}\right)^{2}} e^{-t\left(\tau_{a}+\tau_{p}\right) / \tau_{a} \tau_{p}}+\frac{\tau_{p}^{-1}\left(v \tau_{a}\right)^{2}+3 D}{1+\tau_{a} / \tau_{p}} t+\frac{3 D \tau_{p}-\left(v \tau_{a}\right)^{2}\left(1+2 \tau_{p} / \tau_{a}\right)}{\left(1+\tau_{p} / \tau_{a}\right)^{2}}\right\} .
$$

Over a period of duration $\tau_{a}+\tau_{p}$, during which the directional persistence in the active phase causes a nonlinear time dependence of $\left\langle\left|\mathbf{r}^{2}(t)\right|\right\rangle$ and hence a local violation of the central limit theorem, an effective diffusive regime $\left\langle\left|\mathbf{r}^{2}(t)\right|\right\rangle \simeq t$ is established. Equation (5) is a transcendental equation for $\tau_{i}$, depending on only three parameters: the typical distance covered in the active and passive phases $x_{a}=v \tau_{a}$ and $x_{p}=\sqrt{D \tau_{p}}$, respectively, and the Péclet number Pe $=L v / D$. To estimate the efficiency of active trafficking with respect to diffusion we compare $\tau_{i}$ with the purely passive equilibration time $\tau_{0} \equiv L^{2} / 6 D$. Figures 2(a)-2(d) show results for various Pe values [Fig. 2(e)] typical for biological systems.

Active transport is more efficient for larger particles (small $D$ ) in larger domains, a consequence of the finite motor velocity and instantaneous directionality of active motion. Namely, in terms of the MSD, diffusion and active motion display different time scaling $\left(\simeq t\right.$ versus $\left.\simeq t^{2}\right)$ : Considering only pure passive and active motion for $\mathrm{Pe}<6$ diffusion is more efficient. In the intermittent case the motion has a transient period of duration $\tau_{a}+\tau_{a}$, which corresponds to a parameter-dependent combination of both regimes during the relaxation towards the equilibrium partitioning between phases $a$ and $p$. After this transient period an effective diffusive regime is established with diffusivity $D_{\text {eff }}=\left[D+\left(v \tau_{a}\right)^{2} / 3 \tau_{p}\right] /(1+$ $\left.\tau_{a} / \tau_{p}\right)$, which may or may not be larger than $D$. Thus $\tau_{i}$ can be smaller or larger than $\tau_{0}$. Trafficking of vesicles with $D \lesssim 10^{-2} \mu \mathrm{m}^{2} / \mathrm{s}$ therefore mostly profits from active motion, whereas active motion of smaller proteins with $D \sim 10 \mu \mathrm{m}^{2} / \mathrm{s}$ will only be more efficient over large distances as in eukaryotic cells (especially for neurons) and only if accompanied by significant phases of passive diffusion. The observed features explain why it is profitable for a cell to use active transport 

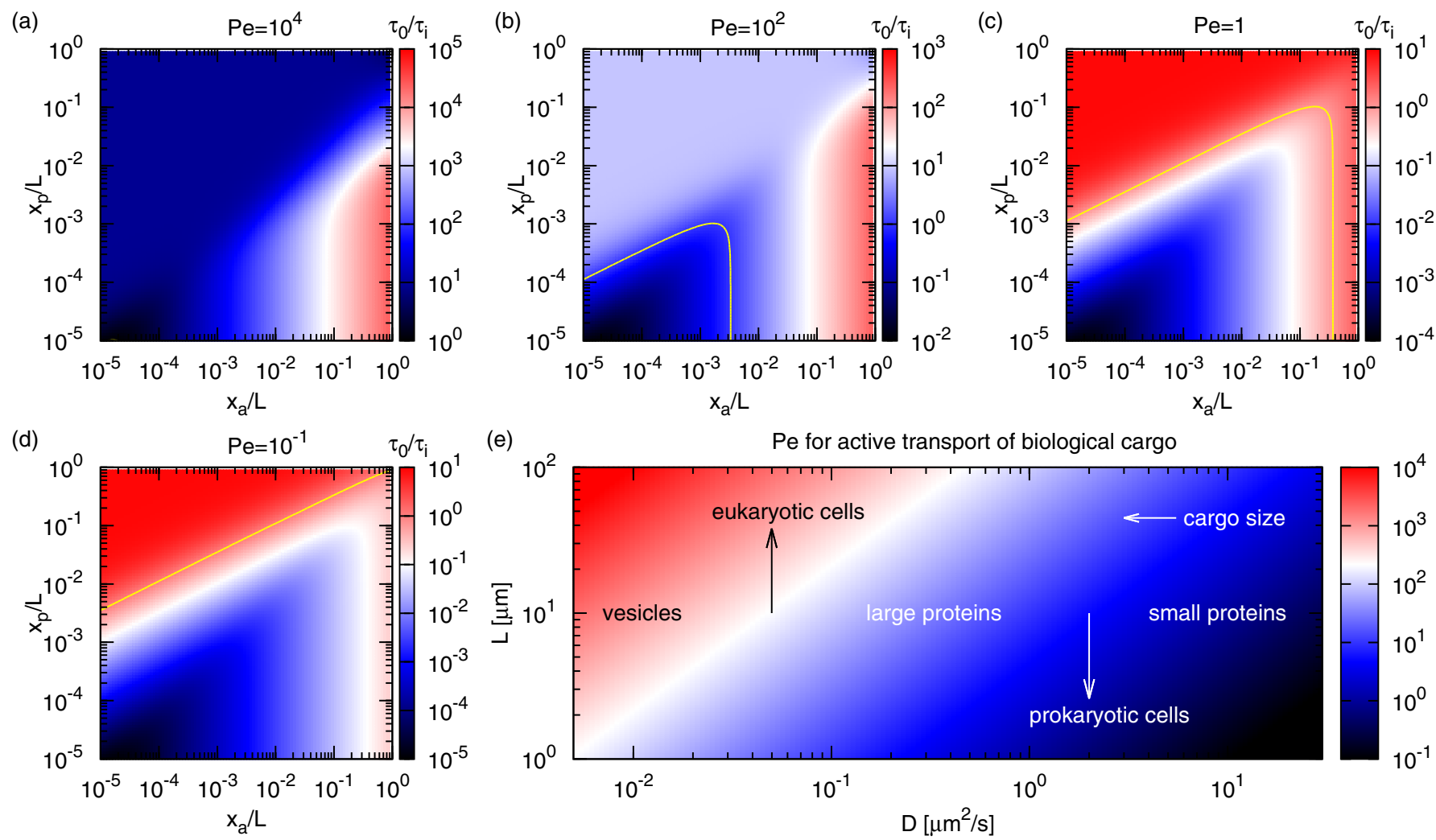

FIG. 2. (Color online) (a)-(d) Equilibration times $\tau_{0} / \tau_{i}$ for various Péclet numbers Pe from numerical inversion of Eq. (5). Yellow lines denote $\tau_{0} / \tau_{i}=1$. When $\tau_{0} / \tau_{i}>1$ active trafficking is more efficient. Note the different scales for $\tau_{0} / \tau_{i}$. (e) The Pe as a function of cell (domain) size $L$ and diffusivity $D$ for biological cells $[19,35]$. Arrows denote increasing particle size from right (small proteins) to left (large vesicles) and cell size from bottom (prokaryotic cells) to top (eukaryotic cells). The border between prokaryotic and eukaryotic cells is around $10 \mu \mathrm{m}$. For Pe $\sim 10^{4}$ (vesicles or large proteins in eukaryotic cells), active transport always improves the speed. For small proteins in eukaryotic cells and large proteins in prokaryotic cells $\left(10 \lesssim \mathrm{Pe} \lesssim 10^{2}\right)$, in addition to large $x_{a}$ a large $x_{p}$ is necessary for active transport to be more efficient. For Pe $\lesssim 10^{-1}$ active transport is always less efficient.

for trafficking of larger particles [18,36], despite demanding more cellular resources. Similarly, active diagnostics [13] can be faster and hence allow for a higher throughput.

\section{PRECISION}

Since the precision of the receptor measurement of the signaling molecule concentration should be maximized, we consider small deviations from the equilibrium values $n=$ $\langle n\rangle+\delta n$ and $c_{p, a}=\left\langle c_{p, a}\right\rangle+\delta c_{p, a}[1]$, taking into account the detailed balance for the binding-unbinding transitions via $k_{\text {on }}\left\langle c_{p}\right\rangle / k_{\text {off }}=\exp \left(F / k_{B} T\right)$, where $F$ is the binding free energy. Detailed balance requires $\delta k_{\text {on }} / k_{\text {on }}-\delta k_{\text {off }} / k_{\text {off }}=$ $\delta F / k_{B} T$. The different prefactors of Refs. [1,11] are not relevant as we consider precision ratios differing at most by a factor $\sim O(1)$ [20]. Solving Eqs. (2) and (3), we obtain [20]

$\delta \tilde{n}(\omega)=\frac{k_{\mathrm{off}}\langle n\rangle \beta \delta \tilde{F}(\omega)}{\left.\tau_{c}^{-1}-i \omega\left(1+\left[k_{\mathrm{on}}(1-\langle n\rangle) /(2 \pi)^{3}\right]\right) \int d \mathbf{k} / \Xi(\mathbf{k}, \omega)\right)}$,

where $\beta=1 / k_{B} T$ and we defined the correlation time $\tau_{c} \equiv$ $\left(k_{\text {on }}\left\langle c_{p}\right\rangle-k_{\text {off }}\right)^{-1}$ for two-state Markovian switching. The auxiliary functions are defined as

$$
\begin{aligned}
& \Xi(\mathbf{k}, \omega) \equiv-i \omega+D k^{2}+\tau_{p}^{-1}-\left(4 \pi \tau_{p} \tau_{a}\right)^{-1} \Psi(\mathbf{k}, \omega), \\
& \Psi(\mathbf{k}, \omega) \equiv \int_{0}^{\pi} \int_{0}^{2 \pi} \frac{d \theta \sin (\theta) d \varphi}{\tau_{a}^{-1}-i[\omega-\mathbf{v}(\theta, \varphi) \cdot \mathbf{k}]} .
\end{aligned}
$$

Note that the linearized equation for $\delta n(t)$ exactly obeys the generalized Langevin equation

$$
\int_{0}^{t} d t^{\prime} \gamma\left(t-t^{\prime}\right) \frac{d \delta n\left(t^{\prime}\right)}{d t^{\prime}}+\tau_{c}^{-1} \delta n(t)=\beta k_{\mathrm{off}}\langle n\rangle \delta F(t)
$$

with the kernel $\gamma(t)=\delta\left(t-t^{\prime}\right)+\mathcal{F}^{-1}\left\{\int\left[d \mathbf{k} k_{\text {on }}(1-\langle n\rangle) /\right.\right.$ $\left.\left.(2 \pi)^{3} \Xi(\mathbf{k}, \omega)\right]\right\}$, where $\mathcal{F}^{-1}$ denotes the inverse Fourier transform $\omega \rightarrow t$. Being interested in the low-frequency limit $\Psi(\mathbf{k}, \omega) \simeq \Psi(\mathbf{k}, 0)$, the integral over $\varphi$ in $\Psi(\mathbf{k}, \omega)$ can be evaluated as a contour integral along the unit circle via the method of residues, while the second one directly leads to

$$
\Psi(\mathbf{k}, 0)=(4 \pi / v k) \tan ^{-1}\left(v \tau_{a} k\right)
$$

with $k \equiv|\mathbf{k}|$. Using Eq. (9), we obtain $\delta \tilde{n}(\omega)$ as a function of $\delta \tilde{F}(\omega)$ explicitly. The linear response function of the receptor occupancy (the coordinate) to a change in the free-energy difference between bound and freely diffusing species (the thermodynamically conjugate force) is then $\delta \tilde{n}(\omega) / \delta \tilde{F}(\omega)$ and is related to the power spectrum of $\delta n$ via the fluctuation- 
dissipation theorem $S_{n}(\omega)=2 / \beta \omega \operatorname{Im}[\delta \tilde{n}(\omega) / \delta \tilde{F}(\omega)] \quad$ [1]. From Eq. (9) we obtain the Lorentzian low-frequency power spectrum of fluctuations of receptor occupancy

$$
\begin{aligned}
& S_{\delta n}(\omega) \\
& \quad \simeq \frac{2 k_{\mathrm{on}}\left\langle c_{p}\right\rangle(1-\langle n\rangle)\left\{1+\left[k_{\mathrm{on}}(1-\langle n\rangle) / 2 \pi D a\right] \Lambda\left(x_{a}, x_{p}\right)\right\}}{\omega^{2}\left\{1+\left[k_{\mathrm{on}}(1-\langle n\rangle) / 2 \pi D a\right] \Lambda\left(x_{a}, x_{p}\right)\right\}^{2}+\tau_{c}^{-2}},
\end{aligned}
$$

where we cut the integral in the inverse Fourier transform at $\pi / a$ to avoid the UV divergence and introduced

$$
\Lambda\left(x_{a}, x_{p}\right)=\int_{0}^{1} \frac{d q}{1+\left(a / \pi x_{p} q\right)^{2}\left[1-\tan ^{-1}\left(\pi x_{a} q / a\right) a / \pi x_{a} q\right]}
$$

and $\Lambda\left(x_{a}, x_{p}\right) \leqslant 1$ [20]. The error in the receptor occupancy for equilibrium sensing is then $\delta n^{2}=S_{\delta n}(0) / \tau_{m}$,

$$
\delta n^{2}=\frac{2\langle n\rangle[1-\langle n\rangle]}{\left(k_{\mathrm{on}}\left\langle c_{p}\right\rangle-k_{\mathrm{off}}\right) \tau_{m}}\left\{1+\left[k_{\mathrm{on}}(1-\langle n\rangle) / 2 \pi D a\right] \Lambda\left(x_{a}, x_{p}\right)\right\} .
$$

A change in concentration is equivalent to a change in $F$, $\delta c_{p} /\left\langle c_{p}\right\rangle=\beta \delta F$. Using this, it can be shown [1] that $S_{c}(\omega)=$ $\left(\beta\left\langle c_{p}\right\rangle\right)^{2}|\delta \tilde{n}(\omega) / \delta \tilde{F}(\omega)|^{-2} S_{n}(\omega)$. Within the linear response regime and for receptor measurement times $\tau_{m}$ exceeding any correlation times, the corresponding relative error for the concentration measurement is

$$
\frac{\overline{\delta c_{p}^{2}}}{\left\langle c_{p}\right\rangle^{2}}=\frac{2}{k_{\mathrm{on}}\left\langle c_{p}\right\rangle(1-\langle n\rangle) \tau_{m}}+\frac{\Lambda\left(x_{a}, x_{p}\right)}{\pi D a\left\langle c_{p}\right\rangle \tau_{m}} .
$$

Compared to passive diffusion [1], our result (13) differs by the factor $\Lambda\left(x_{a}, x_{p}\right)$. Moreover, it can be shown that the steady concentration in the passive phase is given by

$$
\begin{aligned}
\left\langle c_{p}\right\rangle= & \frac{c_{\mathrm{tot}}-V^{-1}}{2\left(1+\tau_{a} / \tau_{p}\right)}-\frac{k_{\mathrm{off}}}{2 k_{\mathrm{on}}}+\left[\left(\frac{c_{\mathrm{tot}}-V^{-1}}{2\left(1+\tau_{a} / \tau_{p}\right)}-\frac{k_{\mathrm{off}}}{2 k_{\mathrm{on}}}\right)^{2}\right. \\
& \left.+\frac{c_{\mathrm{tot}} k_{\mathrm{off}}}{k_{\mathrm{on}}\left(1+\tau_{a} / \tau_{p}\right)}\right]^{1 / 2},
\end{aligned}
$$

where $V$ is the volume of the cell or its domain. In addition, the relation between $\langle n\rangle$ and $\left\langle c_{p}\right\rangle$ is given by $\langle n\rangle=(1+$ $\left.k_{\text {off }} / k_{\text {on }}\left\langle c_{p}\right\rangle\right)^{-1}$. This means that any change in $\tau_{a} / \tau_{p}$ at constant $c_{\text {tot }}$ also changes $\langle n\rangle$. Since the receptor directly measures $\langle n\rangle$ the above equation for $\langle n\rangle$ proves that there is no unique or natural way of gauging the sensing precision for active motion versus passive diffusion alone. Below we therefore focus on selected limiting cases. Note that our mean-field treatment neglects nonequilibrium fluctuations on the level of single molecular motors [37]. Active motion thus effects a chemical reaction randomly removing and adding molecules to the passive phase and thus allows the use of the fluctuation-dissipation theorem. Moreover, the equilibrium sensing precision is fundamentally different from the nonequilibrium intermittent search kinetics [32] as the former requires equilibrium partitioning between $a$ and $p$ phases [20].

We now focus on the transport controlled regime in which $k_{\text {on }}\left\langle c_{p}\right\rangle, k_{\text {off }} \gg \tau_{a}^{-1}, \tau_{p}^{-1}$ and $k_{\text {on }}\left\langle c_{p}\right\rangle / k_{\text {off }} \gg 1$ and obtain $\left\langle c_{p}\right\rangle=c_{\text {tot }} /\left(1+\tau_{a} / \tau_{p}\right)$. For convenience we absorbed the term $1 / V$ in the total concentration $c_{\text {tot }}-V^{-1} \rightarrow c_{\text {tot }}$. In this regime we neglect the first term in Eq. (13) and consider the remaining active noise floor. If the active excursions are short compared to the receptor size $x_{a} / a \ll 1$,

$$
\begin{aligned}
& \Lambda\left(x_{a}, x_{p}\right) \sim \frac{\sqrt{5} a x_{p}}{\left(\pi x_{a}\right)^{2}} \\
& \times \frac{\tanh ^{-1}\left\{\left[\left(\pi x_{a}\right)^{2} / \sqrt{5} a x_{p}\right]\left[1+(1 / 3)\left(x_{a} / x_{p}\right)^{2}\right]^{-1 / 2}\right\}}{\left[1+(1 / 3)\left(x_{a} / x_{p}\right)^{2}\right]^{1 / 2}} .
\end{aligned}
$$

In the biologically more important situation $x_{a} \gg a$,

$$
\Lambda\left(x_{a}, x_{p}\right) \sim 1-\tan ^{-1}\left(\pi x_{p} / a\right) /\left(\pi x_{p} / a\right),
$$

which has the lower bound $\Lambda_{\min } \sim\left(\pi x_{p} / a\right)^{2}\left[1-\left(\pi x_{p} / a\right)^{2}\right]$ as $x_{p} / a \rightarrow 0$. This might suggest an approach towards an infinite absolute precision of the transport term as $x_{p} \rightarrow$ 0 . However, at fixed total concentration $c_{\text {tot }}$ of signaling particles in the transport controlled regime we have that $\left\langle c_{p}\right\rangle=$ $c_{\text {tot }} /\left(1+\tau_{a} / \tau_{p}\right)$ corresponding to $\left\langle c_{p}\right\rangle \rightarrow 0$ as $x_{p} \rightarrow 0$, hence diverging relative fluctuations. Therefore, there still exists a noise floor to active sensing, but it can be significantly reduced as explained below.

We gauge the sensing precision at equal $c_{\text {tot }}$ and equal $\left\langle c_{p}\right\rangle$, corresponding to the lower and upper bounds for the gain of active focusing [20]. At equal $c_{\text {tot }}$ we compare the relative accuracy of measuring different concentrations of freely diffusing molecules in active and passive sensing, finding that the precision is always worse for active transport as compared to free diffusion [Figs. 3(a)-3(c)] and asymptotic results in Figs. 3(e) and 3(f), becoming worse with longer active excursions: Despite reducing the absolute fluctuations by active dynamics, we are measuring smaller and smaller effective concentrations. Conversely, if we compare the precision at equal $\left\langle c_{p}\right\rangle=c_{\text {tot }}\left(1+\tau_{a} / \tau_{p}\right)$ of free molecules [Figs. 3(d)-3(f)] the accuracy can be improved significantly as long as $x_{p} \lesssim a$ (otherwise, in this regime active trafficking does not affect sensing precision). The counting noise is reduced since (i) only fluctuations on a scale $\sim a$ are relevant for the sensing accuracy and (ii) the receptor only recognizes free particles. Hence, at finite temperatures perfect signaling occurs when upon release from the receptor the particles immediately bind to a motor and are swept away over distances greater than $a$. Concurrently diffusive displacements must be $\lesssim a$ to assure focused delivery such that any unbinding form the motor only contributes if it occurs at $\left|\mathbf{r}-\mathbf{r}_{0}\right| \lesssim a$. This reduction of local concentration fluctuations via intermittent active excursions is exactly our active signal focusing.

If instead we assume that the total concentration can be extracted immediately from the measured receptor occupancy, the error in measuring $c_{\text {tot }}$ follows from $\delta c_{\text {tot }}^{2}=$ $\left(d c_{\text {tot }} / d\left\langle c_{p}\right\rangle\right)^{2}\left(d\left\langle c_{p}\right\rangle / d\langle n\rangle\right)^{2} \delta n^{2}$, yielding the precision ratio [20]

$$
\frac{\delta c_{\mathrm{tot}}^{2}}{\delta c_{\mathrm{tot}, 0}^{2}}=\left[\frac{\left(1+\tau_{a} / \tau_{p}\right)^{-1}+k_{\mathrm{on}} c_{\mathrm{tot}} / k_{\mathrm{off}}}{1+k_{\mathrm{on}} c_{\mathrm{tot}} / k_{\mathrm{off}}}\right]^{2}\left(1+\frac{\tau_{a}}{\tau_{p}}\right) \Lambda\left(x_{a}, x_{p}\right) .
$$



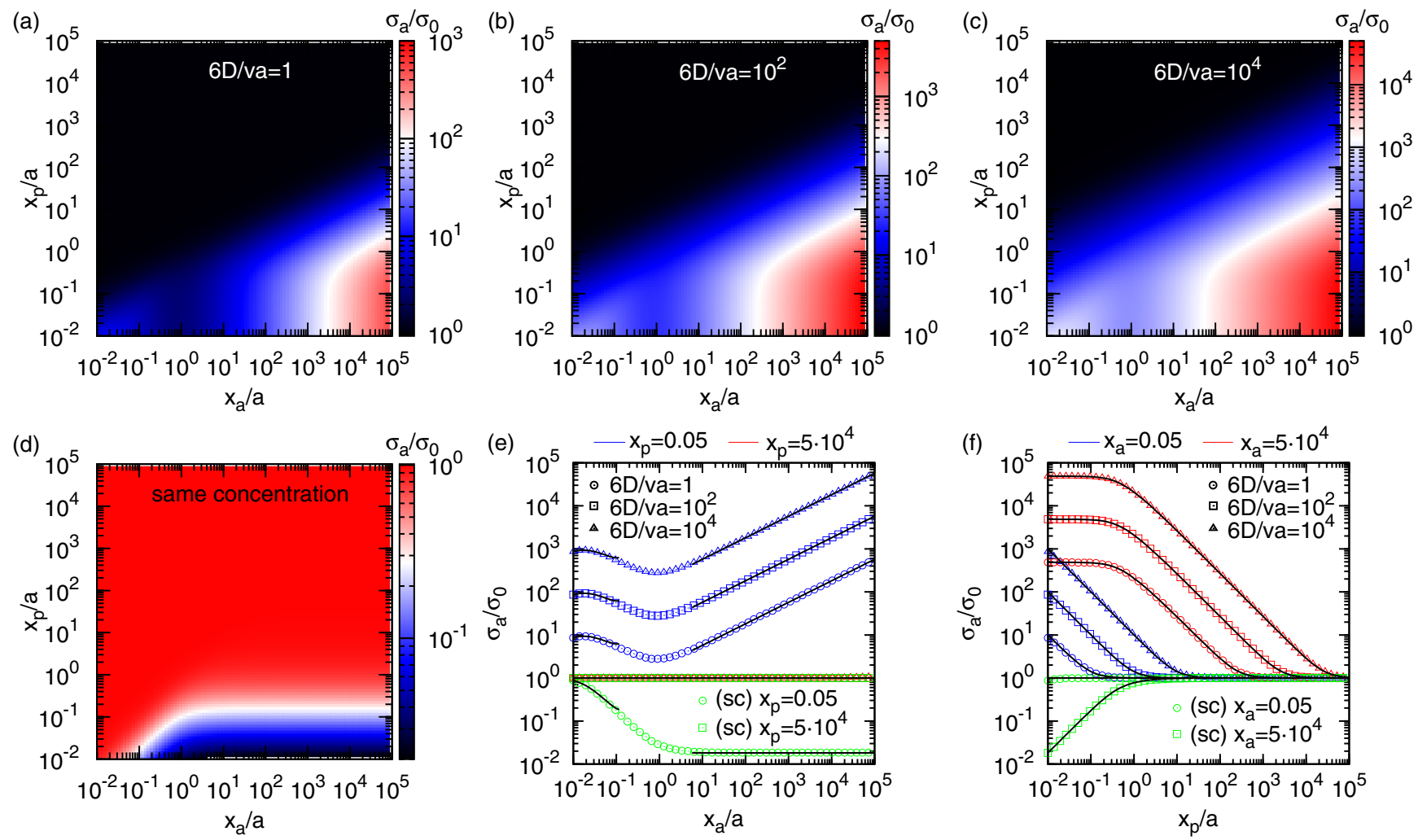

FIG. 3. (Color online) (a)-(c) Fractional variance $\sigma_{a}=\left[\overline{\delta c_{p}^{2}} /\left\langle c_{p}\right\rangle^{2}\right]^{1 / 2}$ of concentration fluctuations at the receptor site for active trafficking compared to thermal diffusion $\sigma_{0}=\left[\pi D a c_{\mathrm{tot}} \tau_{m}\right]^{-1 / 2}$ as a function of the average distance traveled in both phases in units of the receptor radius $a$. In (a)-(c) the total concentration $c_{\text {tot }}$ is kept constant, hence decreased sensing accuracy is solely due to exceedingly small equilibrium concentrations in the passive phase. (d) $\sigma_{a} / \sigma_{0}$ for equal equilibrium concentrations [sc in (e) and (f)] in the passive phase $\left\langle c_{p}\right\rangle$ for different total concentrations equal to $c_{\text {tot }}\left(1+\tau_{a} / \tau_{p}\right)$ : A strong increase in sensing accuracy is observed for $x_{p} / a \rightarrow 0$. (a)-(d) use the results (13) and (S8) in [20]. (e) Horizontal cross sections of $\sigma_{a} / \sigma_{0}$ at large and small $x_{p}$ (symbols) compared to the approximations (15) and (16). (f) Vertical cross sections at large and small $x_{a}$ (symbols) compared to the approximations (15) and (16). Note the different scales for $\sigma_{a} / \sigma_{0}$ and the excellent agreement between full and approximate results. The nonmonotonicity at small $x_{p}$ is due to the interplay of signal focusing (smaller $\Lambda$ ) and decreasing $\left\langle c_{p}\right\rangle$.

Equation (16) for $x_{a} \gg a$ and $\tau_{a} \gg \tau_{p}$ then leads to

$$
\frac{\delta c_{\mathrm{tot}}^{2}}{\delta c_{\mathrm{tot}, 0}^{2}} \simeq \frac{D x_{a}}{v x_{p}^{2}}\left(1-\frac{\tan ^{-1}\left(\pi x_{p} / a\right)}{\pi x_{p} / a}\right) .
$$

In this limit, signal focusing always allows more precise sensing as long as $D \tau_{a} / a^{2}<1 / \pi^{2}$. Alternatively, involving receptor binding from the active phase, e.g., via active cytoplasmic drag, we recover Eq. (1) but with $D_{\text {eff }}$ defined above. Here active signaling is always beneficial for $\frac{x_{a}}{x_{p}} \geqslant \frac{3 D}{v x_{p}}$ and can become infinitely precise [20].

\section{CONCLUSION}

The past decade has seen significant activity to explore the counting noise for purely diffusive scenarios [1-11] and to analyze the speedup of receptor binding due to the topological coupling of one- and three-dimensional diffusion for gene regulation in the facilitated diffusion model $[12,38]$. Here we extend this approach and assess the counting noise experienced by local concentration measurements of signaling particles in the presence of an active transport component. This occurs for various signaling cues (proteins, mRNA molecules, vesicles containing signaling molecules, or viruses) by direct shuttling by molecular motors or by cytoplasmic drag.

Compared to the purely diffusive signaling considered so far, we showed that the counting noise for active sensing can become significantly reduced due to active focusing. The only contributions to the counting noise stem from particles, which are actively transported to within the particle's typical freediffusion distance to the receptor. This reduces the correlation time of the receptor occupancy noise and renders the averaging over a measurement time $\tau_{m}$ more efficient. Conversely, when the receptor can also bind molecules in the active phase, the noise reduction is due to an increased effective diffusivity. The importance of active signaling in cellular regulation is well recognized $[33,39]$. In agreement with our results, in biological systems active transport is indeed employed to move larger particles (e.g., vesicles or viruses) with intrinsically small $D[19,23-25,28,36]$. As a result, even during longer periods of detachment from motors these particles barely move [18,2325,36]. The typical experimental values $x_{a} \simeq 0.5-10 \mu \mathrm{m}$ [24-26,36] for $a \simeq 1-10 \mathrm{~nm}$ in fact fulfill the requirements of our model for signal focusing. However, as discussed here, also smaller particles such as mRNA and proteins experience active motion components [29,30], effecting active focusing for their detection. In living cells the motor tracks are often 
not ideally disordered, as assumed here, but biased towards the receptor [39]. An expected net directional bias towards the receptor, while not impeding signal focusing as long as $x_{a} \gg a$ and $x_{p} \lesssim a$, would enhance the rate of delivery and simultaneously increase the local concentration $\left\langle c_{p}\left(\mathbf{r}_{0}\right)\right\rangle$ at the receptor for equal $c_{\text {tot }}$. Signal focusing is thus inherent to active cellular signaling. Conversely, despite the great technological advance over the years, molecular motor-powered diagnostic devices have not yet demonstrated a performance beyond the existing passive techniques, but a large superiority is much anticipated [13]. Our results confirm such expectations and present a theoretical basis for their systematic improvement and development. Our intermittent active signaling model complements approaches describing signaling in the presence of a continuous active hydrodynamic flow of the cellular actomyosin cortex [33].

\section{ACKNOWLEDGMENTS}

We thank Andrey Cherstvy, Olivier Bénichou, and Raphael Voituriez for discussions. We acknowledge funding through an Alexander von Humboldt Fellowship and ARRS Program No. P1-0002 (A.G.) and an Academy of Finland FiDiPro grant (R.M.).
[1] W. Bialek and S. Setayeshgar, Proc. Natl. Acad. Sci. USA 102, 10040 (2005).

[2] H. C. Berg and E. M. Purcell, Biophys. J. 20, 193 (1977).

[3] W. Bialek and S. Setayeshgar, Phys. Rev. Lett. 100, 258101 (2008).

[4] R. G. Endres and N. S. Wingreen, Proc. Natl. Acad. Sci. USA 105, 15749 (2008).

[5] W.-J. Rappel and H. Levine, Phys. Rev. Lett. 100, 228101 (2008).

[6] G. Tkačik, T. Gregor, and W. Bialek, PLoS ONE3, e2774 (2008).

[7] B. Hu, W. Chen, W.-J. Rappel, and H. Levine, Phys. Rev. Lett. 105, 048104 (2010).

[8] K. Wang, W.-J. Rappel, R. Kerr, and H. Levine, Phys. Rev. E 75, 061905 (2007).

[9] B. Hu, D. A. Kessler, W.-J. Rappel, and H. Levine, Phys. Rev. Lett. 107, 148101 (2011).

[10] C. C. Govern and P. R. ten Wolde, Phys. Rev. Lett. 109, 218103 (2012).

[11] C. Kaizu et al., Biophys. J. 106, 976 (2014).

[12] G. Tkačik and W. Bialek, Phys. Rev. E 79, 051901 (2009).

[13] T. Korten, A. Månsson, and S. Diez, Curr. Opin. Biotechnol. 21, 477 (2010); H. Hess and V. Vogel, Rev. Mol. Biotechnol. 82, 67 (2001).

[14] G.-W. Li and X. S. Xie, Nature (London) 475, 308 (2011).

[15] J. C. M. Gebhardt et al., Nat. Methods 10, 421 (2013).

[16] F. Persson, M. Lindén, C. Unoson, and J. Elf, Nat. Methods 10, 265 (2013).

[17] P. Hammar, M. Walldén, D. Fange, F. Persson, O. Baltekin, G. Ullman, P. Leroy, and J. Elf, Nat. Genet. 46, 405 (2014).

[18] W. Bialek, Biophysics: Searching for Principles (Princeton University Press, Princeton, 2012).

[19] B. Alberts et al., Molecular Biology of the Cell (Garland, New York, 2002).

[20] See Supplemental Material at http://link.aps.org/supplemental/ 10.1103/PhysRevE.92.010701 for calculations, full results for the variance of the total concentration estimate as well as for the precision with combined receptor recognition and for an estimate of the difference in prefactors.

[21] A. B. Kolomeisky and M. E. Fischer, Annu. Rev. Phys. Chem. 58, 675 (2007).

[22] F. Jülicher, A. Ajdari, and J. Prost, Rev. Mod. Phys. 69, 1269 (1997).

[23] H. Salman et al., Biophys. J. 89, 2134 (2005).

[24] S. Huet, E. Karatekin, V. S. Tran, S. Cribier, and J. P. Henry, Biophys. J. 91, 3542 (2006).

[25] A. Vermehren-Schmaedick et al., PLoS ONE 9, e95113 (2014).
[26] D. Arcizet, B. Meier, E. Sackmann, J. O. Rädler, and D. Heinrich, Phys. Rev. Lett. 101, 248103 (2008).

[27] G. J. Doherty and H. T. McMahon, Annu. Rev. Biochem. 78, 857 (2009); L. M. Traub, Nat. Rev. Mol. Cell Biol. 10, 583 (2009); A. Sorkin and M. von Zastrow, ibid. 10, 609 (2009).

[28] G. Seisenberger, M. U. Ried, T. Endreß, H. Büning, M. Hallek, and C. Bräuchle, Science 294, 1929 (2001).

[29] D. St. Johnston, Nat. Rev. 6, 363 (2005); H. Tekotte and I. Davis, Trends Genet. 118, 636 (2002); D. Fusco et al., Curr. Biol. 13, 161 (2003); R. D. Vale, Cell 112, 467 (2003).

[30] M. Mussel, K. Zeevy, H. Diamant, and U. Nevo, Biophys. J. 106, 2710 (2014); S. Roy et al., J. Neurosci. 27, 3131 (2007); D. A. Scott et al., Neuron 70, 441 (2011).

[31] O. Bénichou, C. Loverdo, M. Moreau, and R. Voituriez, Rev. Mod. Phys. 83, 81 (2011).

[32] C. Loverdo, O. Bénichou, M. Moreau, and R. Voituriez, Nat. Phys. 9, 134 (2008); J. Stat. Mech. (2009) P02045.

[33] N. W. Goehring et al., Science 334, 1137 (2011); J. S. Bois, F. Jülicher, and S. W. Grill, Phys. Rev. Lett. 106, 028103 (2011); K. V. Kumar, J. S. Bois, F. Jülicher, and S. W. Grill, ibid. 112, 208101 (2014).

[34] The molecular motor speed $|\mathbf{v}(\Omega)| \sim 1 \mu \mathrm{m} / \mathrm{s}$ is widely independent of the particle size [19].

[35] P. C. Bressloff and J. M. Newby, Rev. Mod. Phys. 85, 135 (2013).

[36] N. Hirokawa, Y. Noda, Y. Tanaka, and S. Niwa, Nat. Rev. Mol. Cell Biol. 10, 682 (2009); C. Desnos and S. Huet, Biol. Cell. 99, 411 (2007).

[37] J. Prost, J.-F. Joanny, and J. M. R. Parrondo, Phys. Rev. Lett. 103, 090601 (2009); L. Dinis, P. Martin, J. Barral, J. Prost, and J. F. Joanny, ibid. 109, 160602 (2012).

[38] G. Kolesov et al., Proc. Natl. Acad. Sci. USA 104, 13948 (2007); M. A. Lomholt et al., ibid. 106, 8204 (2009); E. F. Koslover, M. A. Díaz de la Rosa, and A. J. Spakowitz, Biophys. J. 101, 856 (2011); M. Bauer and R. Metzler, PLoS ONE 8, e53956 (2013); Biophys. J. 102, 2321 (2012); M. Bauer, E. S. Rasmussen, M. A. Lomholt, and R. Metzler, Sci. Rep. 5, 10072 (2015); O. Pulkkinen and R. Metzler, Phys. Rev. Lett. 110, 198101 (2013); C. A. Brackley, M. E. Cates, and D. Marenduzzo, ibid. 111, 108101 (2013); 109, 168103 (2012); T. Hu, A. Y. Grosberg, and B. Shklovskii, Biophys. J. 90, 2731 (2006); A. B. Kolomeisky and A. Veksler, J. Chem. Phys. 136, 125101 (2012); A. Marcovitz and Y. Levy, Proc. Natl. Acad. Sci. USA 108, 17957 (2011); see also the different scenario in J. Paijmans and P. R. ten Wolde, Phys. Rev. E 90, 032708 (2014).

[39] B. J. Schnapp, J. Cell Sci. 116, 2125 (2003). 